\title{
La radiación ultravioleta y su incidencia en la ciudad de Chimbote*
}

Genaro Uribe Santos**

http://dx.doi.org/10.21503/lex.v9i8.415

* Ponencia presentada en el Primer Congreso Internacional de Medio Ambiente y Derecho Ambiental, los días 26, 27 y 28 de mayo de 2011, en la Universidad Alas Peruanas Filial Chimbote.

** Abogado, posgrado en Medio Ambiente, Recursos Hídricos, Desarrollo Agrícola y Sostenible por la Universidad de Cuyo y del Sur de Bahía Blanca de Argentina; Maestría en Derecho Ambiental por la Universidad Politécnica de Barcelona de España, docente universitario, Presidente de la Academia Transdisciplinaria Internacional del Ambiente (ATINAA).

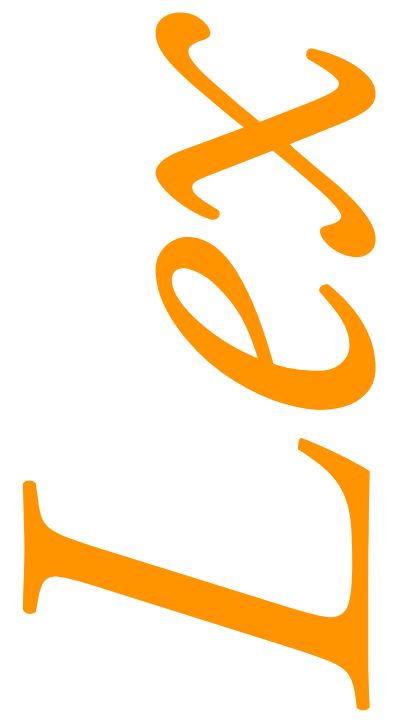




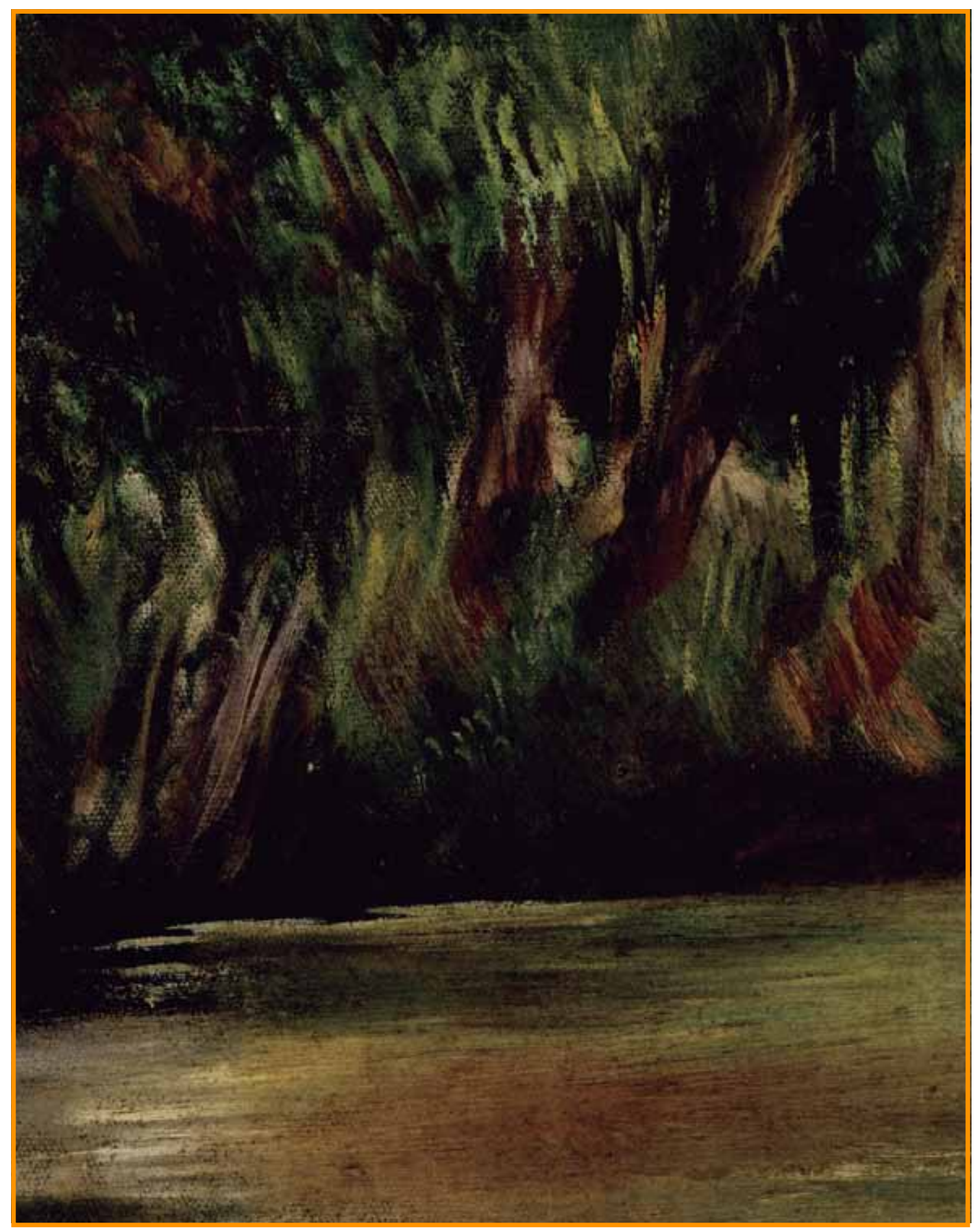


Tomo todos sabemos, los espectros electromagnéticos que irradia el Sol producen ondas de energía de diferente intensidad que van desde el radio (onda larga) hasta los rayos gamma (onda corta). Estas ondas de energía llegan al planeta Tierra, afectándolo de manera directa pero en diferente modo, según la forma en que estas toquen la corteza terrestre, siendo en el caso de los polos de manera tangencial (chocan en la corteza y luego rebotan), mientras que en el cinturón ecuatorial es de manera perpendicular (choca de manera frontal).

Esta llegada perpendicular de la energía solar genera una especial incidencia sobre todo en nuestra región geográfica, pues prácticamente implica que el Perú se vea afectado casi en su totalidad y todos los meses del año por la radiación ultravioleta (o R-UV), siendo las zonas del sur, especialmente Arequipa, Moquegua, Cuzco y Tacna las de mayor incidencia, las del centro, como Lima, Ancash, La Libertad y Tumbes de incidencia mediana y algunas zonas de Loreto y Madre de Dios de poca incidencia, dependiendo especialmente de la estación en la que se encuentren.

En general, el Perú está siempre bajo los efectos de la radiación ultravioleta. En el caso de Chimbote, sólo algunos meses, en especial en octubre, la radiación es mínima. Sin embargo, al promediar el mes de noviembre, aumenta de manera vertiginosa en casi todo el Perú, afectando también a esta ciudad de forma ineludible, recomenzando así un nuevo ciclo que se prolonga todos los meses hasta un breve descanso en el siguiente decimo mes.

Ahora bien, no debemos perder de vista las condiciones atmosféricas en las que se encuentra sumido nuestro planeta a causa del agujero en la capa de ozono producido por factores antropogénicos, que si bien ha sido declarado en franco retroceso, seguirá ocasionando complicaciones respecto a la R-UV mientras no se hay verificado su cierre total que -según los cálculos de los estudiosos- podría suceder alrededor del año 2025.

Como se sabe, la principal función de la capa de ozono es justamente filtrar la llegada agresiva de la R-UV a nuestro planeta, permitiendo que pasen solo una pequeña cantidad 
de ondas que mantendrán la Tierra en un clima agradable y propicio para la vida, siendo las demás rechazadas hacia el espacio por la reflexión. Cuando la capa de ozono presenta estos agujeros, ya sea por causas antropogénicas o por fenómenos naturales, se suceden en nuestro planeta una serie de fenómenos que alteran nuestra forma de vida, siendo el calentamiento global una de las principales afectaciones.

Es de precisar que estos agujeros no son una suerte de "cascarones de huevos rotos", no están fijos, no son estables, sino por el contrario, son daños a una capa fina que cubre todo el planeta Tierra, que tiene promedio un ancho de 10 kilómetros (algo así como si a una pelota de basquetbol le pasáramos una capa de barniz) y que por su condición de gas, suele moverse en diferentes posiciones, dependiendo mucho del clima y los vientos. Fotografías tomadas a la zona antártica, que es donde se encuentra unos de los agujeros más grandes en la capa de ozono, demuestras que este se mueve en diferentes direcciones, habiendo incluso llegado en algunas ocasiones hasta la zona sudamericana, más propiamente por Argentina, Uruguay, Chile, Bolivia y Perú.

Sudamérica, África y Australia son continentes que tienen incidencia directa de la R-UV sobre sus zonas geográficas, toda vez que se encuentran en el cinturón ecuatorial y por ello la reciben de manera perpendicular, habiéndose observado que la mayor parte de estas afectaciones se ubican (por el lado sudamericano) en casi todo el Perú, especialmente en la zona centro y sur, en la zona norte y centro de Chile, en los países de Angola, Namibia, Botswana y Sudáfrica (por el lado de África), y en las ciudades de Wyndham, Derby, Dampler y Geraldton (en Australia). Una razón para que la incidencia de la R-UV sea especialmente alta en estas regiones, son las características desérticas de las mismas, lo que obviamente potencia la manera en la cual esta radiación llega a la corteza terrestre.

Estudios desarrollados a cargo de la NASA norteamericana han definido que los picos más altos de R-UV se producen en el Perú, muy por encima de los valores clasificados en la escala de radiación ultra violeta que publicara el organismo Mundial de la Salud - OMS.

Según la revista "Expert Review in Molecular Medicine", de la Universidad de Cambridge, la R-UV tiene dos fases que la caracterizan: la positiva y la negativa. La R-UV "positiva" permite eliminar de nuestra atmósfera una serie de gérmenes patógenos que de otro modo tendrían rápida reproducción, poniendo en especial riesgo la salud y subsistencia de la raza humana en el planeta; induce la síntesis de la vitamina $\mathrm{D}$, que ayuda a un mejor estado de salud de las partes óseas de las personas; sirve para tratar ciertos desórdenes en la salud humana, como por ejemplo la psoriasis.

Pero en su faz "negativa", la sobreexposición a la R-UV puede producir quemaduras y bronceados, así como el foto envejecimiento de la piel, inmuno supresión, daños en el DNA, 
mutaciones genéticas, proliferación celular anormal y cánceres de todo tipo, especialmente el de piel.

Decíamos hace un momento que los agujeros en la capa de ozono se producen por efectos antropogénicos, pero también pueden ser por factores naturales. Justamente, el Laboratorio de Física de la Atmósfera del Instituto de Investigaciones Físicas de la Universidad Mayor de San Andrés de La Paz, Bolivia, realizó en 1995 el hallazgo de lo que se ha denominado "Anomalía Sudamericana de la Capa de Ozono", que es en suma un agujero producido por factores naturales que quizás haya acompañado a nuestra región desde la época de la creación de nuestro planeta.

Este agujero abarca casi todo Perú y Bolivia, gran parte del norte chileno y grandes extensiones del norte argentino. Y, al parecer, a diferencia de los otros agujeros en la capa de ozono, generados por obra de las acciones del hombre, esta falla será muy difícil de recuperarse, sino imposible, ocurriendo que estará con nosotros quizás hasta cuando se desaparezca la vida sobre la faz de la Tierra.

Por esta razón, es que especialmente para el Perú y Bolivia la escala del índice de R-UV dado por el OMS no sirve, debido a que los picos en nuestras regiones alcanzan hasta grados mayores a 16, según la magnitud usada por esa misma escala, mientras que las magnitudes que esta define solo llega al nivel 11.

Hablando de valores extremos, se ha estudiado que en Bolivia, por ejemplo, en los veranos de 2002 y 2003 el promedio de R-UV fue de 16, mientras que en varios momentos del año se tuvo picos que sobrepasaban los 22 y 23. En el caso peruano, no se tiene evaluaciones claras al respecto, pero el SENAMHI a dicho en varias ocasiones que, en la media en el Perú, se tiene un índice de R-UV en el promedio "alto" (9-11) de la escala del OMS.

Como hemos mencionado, la sobreexposición a la R-UV en el Perú ha propiciado que especialmente las enfermedades se vean potenciadas por este fenómeno, siendo las oculares una de las principales. Por ejemplo, la catarata senescente, que es la primera causa de ceguera en el país, con el $60 \%$ sobre el total, tiene su génesis en la R-UV. El pterigion, que es una enfermedad que en nuestra realidad se conoce como la "carnosidad" que apare en la cornea del ojo y que suele ser también motivo de daños a la salud de la vista, se origina en la R-UV. Fíjese en este caso que Australia tiene la cabecera con 44\% en el mapa mundial de distribución de esta enfermedad, mientras que Perú tiene el 31\%. Y ambas regiones geográficas están justamente en el cinturón ecuatorial que recibe de manera perpendicular la R-UV.

El cáncer basocelular (cáncer a la piel), que en la mayoría de los casos se origina por sobre exposición de la R-UV, es otra de las afectaciones de esta radiación y constituyen, según 
estadísticas publicadas el 2001 por el Instituto Nacional de Estadística e Informática - INEI, el cuarto en importancia a nivel nacional.

Por otro lado, es muy frecuente pensar que podemos esquivar la R-UV con sólo cobijarnos debajo de la sombra o cuando el cielo está nublado, no teniendo en cuenta que en estos casos, si bien la radiación es amortiguada, no deja de surtir efectos sobre la salud de las personas. Por ejemplo, cuando las nubes no son densas, más del $90 \%$ de la R-UV se filtra entre ellas y llega a la corteza terrestre sin ningún impedimento; o cuando estamos debajo de la sombra, solo logramos esquivar el $50 \%$ de la R-UV, mientras que el otro $50 \%$ sigue afectándonos, al igual que estar sumergidos debajo del agua, pues a medio metro de profundidad, sigue llegándonos el 40\% de la R-UV. Se piensa también, de manera errada, que sólo entre las 10 de la mañana y las 4 de la tarde son las únicas horas que afectan la R-UV; si bien en esas horas el efecto es en su potencia mayor, no deja de ser igualmente significativa la radiación en horas distintas a esas.

¿Cómo nos preparamos para enfrentar los efectos nocivos de la R-UV?

Como ya se dijera, la R-UV en su faz moderada es positiva para el ser humano, pero por el lado negativo, es causante de diversas enfermedades y afecciones, en cuyo caso debemos adoptar medidas concretas, alterar ciertas formas y costumbres de vida y moldearnos a la necesidad de evitar los daños que puedan ocasionar la R-UV incontrolada.

Así:

- Debemos hacer del uso del sombrero de ala ancha un hábito por demás ineludible.

- Debemos usar lentes del sol, con apropiada protección contra la R-UV.

- Se tiene que usar ropa y camisas de mangas largas para proteger los brazos, al igual que se protege el resto del cuerpo con la ropa cotidiana.

- En la medida de lo posible se debe preferir siempre la sombra y el uso de la sombrilla.

- El uso del bloqueador solar factor 15 a más debe ser práctica cotidiana aún cuando se crea que no se va a afectar la piel por estar debajo de la sombra o debajo de las nubes. Afortunadamente, los fabricantes locales están produciendo cada vez mejores bloqueadores que se prestan muy bien a esta necesidad.

- Debe evitarse por todo los modos posibles desarrollar actividades debajo del sol entre las 10 am. y las 4 pm. 
- Hay que verificar que todo lo que usemos sea especialmente diseñado para prevenir la afectación de la R-UV.

- Debe protegerse a las poblaciones de riesgo, tales como los niños, los residentes de raza blanca, los veraneantes, los policías de tránsito, los ambulantes, los excursionistas, los profesores de deporte, etc.

- Deben usarse aparatos que protejan la capa de ozono y que suele distinguirse por los emblemas con que se caracteriza esta particularidad.

- Las autoridades deben incidir en promover el cambio en la escala del Índice de R-UV que se usa a nivel internacional dado por el OMS, debiendo actualizarse a los valores sudamericanos.

Pero también "hay cosas" que no debemos hacer, Por ejemplo:

- Que de una vez las autoridades en general prohíban de manera directa los desfiles escolares, militares, institucionales, etc., así como las marchas y protestas entre las $10 \mathrm{am}$. y 4 pm.

- Promover (como afortunadamente viene ocurriendo) el uso del sombrero de ala ancha en los colegios, como sucede actualmente en Australia.

- No generar alarmas innecesarias, pues si bien la R-UV es dañina y puede ocasionar en casos extremos la muerte, nadie cae muerto en las calles por recibir algunos momentos de radiación. El alarmismo ha demostrado que es el peor consejero para tomar decisiones positivas tendientes a la solución de problemas que por el lado de la cautela y mesura suele arreglarse de mejor modo.

- Aprovechar eficientemente los fondos de la cooperación internacional tendientes a una optimización de los recursos y búsqueda eficiente de soluciones.

Finalmente, la situación de la realidad peruana frente a la R-UV es de especial riesgo. Debemos desarrollar una política definida para esta afectación, que involucre a todos los sectores públicos y privados, motivando voluntades y buscando el camino para encontrar acuerdos que permitan desarrollar una serie de programas tendientes a minimizar los efectos nocivos de la R-UV.

Estando a vísperas de una elección presidencial, en donde en la agenda ambiental de los candidatos prácticamente se señala muy poco o casi en cuanto al tema, preocupa sobremanera que no se evidencia un interés claro para enfrentarlo. 


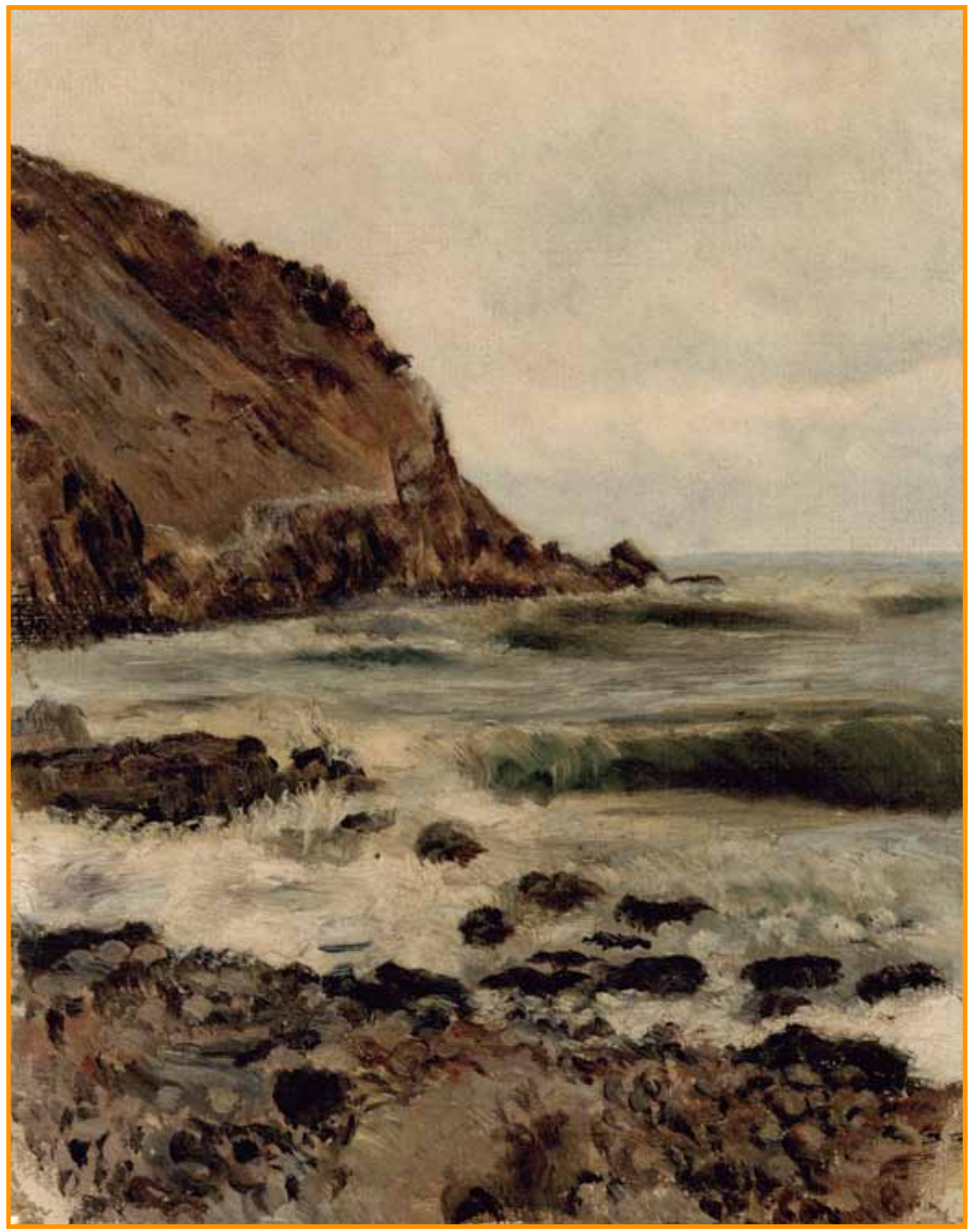

